\title{
CONDITIONS FOR THE EXISTENCE OF CONTRACTIONS IN THE CATEGORY OF ALGEBRAIC SPACES
}

BY

\author{
JOSEPH MAZUR
}

\begin{abstract}
Artin's conditions for the existence of a new contraction of an algebraic subspace are changed. The new conditions are more applicable in special cases, especially when the subspace has a conormal bundle.
\end{abstract}

The precise definition of an algebraic space is rather technical (see [3] or [9]), but we may view algebraic spaces as being local ringed spaces that are locally affine for the étale topology. So schemes are obvious examples of algebraic spaces. However, the category of algebraic spaces is not much larger than that of schemes. For low dimensions there are very few algebraic spaces that are not schemes. In dimension one, every algebraic space (curve) is a scheme; in dimension two, every nonsingular algebraic surface is a scheme [9]. Even in higher dimensions every algebraic space has a dense open subset which is an affine scheme [9].

One nice thing about this new category is that certain constructions (which are not possible in the category of schemes because the Zariski topology is not fine enough) are possible. This paper is concerned with one such constructionthe contraction problem. The contraction problem can be stated as follows: Given a scheme $X$, a closed subscheme $Y$ and a proper map $f: Y \rightarrow Y^{\prime}$, is there a scheme $X^{\prime} \supseteq Y^{\prime}$ and a map $f: X \rightarrow X^{\prime}$ that induces $f$ on $Y$ while being an isomorphism outside $Y$. Castelnuovo studied the classical problem of contracting subschemes to points and gave a criterion for contracting a rational curve on a nonsingular surface to a smooth point [11]. Grauert gave some conditions for the existence of contractions in the category of analytic spaces [6, Satz 8, p. 353]; and showed that in the case where $X$ and $Y$ are smooth with $Y$ of codimension one in $X$, positivity of the conormal bundle is enough to ensure the existence of an analytic contraction.

Artin has given some conditions that ensure the existence of contractions in the category of algebraic spaces [1, Theorem 6.3, p. 121]. His theorem assumes a condition on $I^{n} F / I^{n+1} F$ for every coherent sheaf $F$ on $X$ (where $I$ is the defin- 
ing ideal of $Y$ in $X$ ). The object of this paper is to alter Artin's theorem so as to have a condition that involves only $I / I^{2}$. It turns out that we can reduce our desired condition on $I / I^{2}$ to Artin's condition, so there is no need to reprove Artin's theorem. We do this in Proposition 1. Our result is that if for every coherent sheaf $F$ on $Y$

$$
R^{q} f_{*}\left(S^{n}\left(I / I^{2}\right) \otimes F\right)=0 \quad \text { for } q>0 \text { and } n \gg 0,
$$

then for every coherent sheaf $F$ on $X$ we have

$$
R^{q} f_{*}\left(I^{n} F / I^{n+1} F\right)=0 \text { for } q>0 \text { and } n \gg 0 .
$$

In the case where $Y$ is complete and $I / I^{2}$ is a vector bundle, (1) is just the condition that $I / I^{2}$ be an ample vector bundle (see [8, Chapter $\left.3, \S 1\right]$ ).

Problems that are local for the étale topology are more naturally posed in the category of algebraic spaces. Consider the following question: Given a complete scheme $X$, a closed subscheme $Y$, and an affine Zariski open set $U$ in $Y$, is there an affine Zariski open neighborhood $W$ of $U$ in $X$, such that $U$ is the restriction of $W$ to $Y$ ? The answer is: "In general, no!" However, if we repose the question and ask for a $W$ that is an étale affine neighborhood of $U$ in $X$, the answer is positive. Corollary 2 is precisely what is needed to answer the question. We shall demonstrate the existence of such affine étale neighborhoods in a future paper [10].

I would like to thank Professor Michael Artin for suggesting the topic of this paper. Special thanks go to Jean-François Boutot, who gave the invaluable notation suggestions that appear in the proof of Proposition 1.

All algebraic spaces are assumed to be noetherian.

Proposition 1. Let $X$ be an algebraic space and $Y$ a closed subspace defined by the ideal I of $\mathrm{O}_{X}$. Furthermore, suppose that we have a proper map of algebraic spaces $f: Y \rightarrow Y^{\prime}$ and that for every coherent sheaf $F$ on $Y$ we have

$$
R^{q} f_{*}\left(S^{n}\left(I / I^{2}\right) \otimes F\right)=0 \text { for } q>0 \text { and } n \gg 0
$$

(where $S^{n}\left(I / I^{2}\right)$ is the nth symmetric power of $\left.I / I^{2}\right)$. Then, for every coherent sheaf $F$ on $X$, we have

$$
R^{q} f_{*}\left(I^{n} F / I^{n+1} F\right)=0 \text { for } q>0 \text { and } n \gg 0 .
$$

Proof. We shall introduce some notation and lemmas.

(5) Let $S=\bigoplus_{k \geqslant 0} S^{k}\left(I / I^{2}\right)$.

(6) For every coherent sheaf $F$ on $X$, let 


$$
\operatorname{gr}(F)=\bigoplus_{k \geqslant 0} I^{k} F / I^{k+1} F .
$$

(7) For any graded $S$-Module $E$, let $E_{k}$ denote the homogeneous component of degree $k$.

(8) Let $E$ be a graded $S$-Module. For any integer $n \geqslant 0$, let $E_{k}(n)=E_{n+k}$ and let $E(n)=\bigoplus_{k \geqslant 0} E_{k}(n)$.

We say that $E$ is a coherent $S$-Module, if it is a quasi-coherent $O_{Y}$-Module and an $S$-Module of finite type.

LEMMA 1. If $F$ is a coherent $O_{X^{-M o d u l e}}$, then $\operatorname{gr}(F)$ is a coherent $S$-Module generated over $S$ by its component of degree zero.

Proof. We may assume that $Y$ is affine $(Y=\operatorname{Spec} A)$ and describe the canonical structure of $\operatorname{gr}(F)$ as an $S$-Module. The canonical homomorphism of $A$ Modules $I / I^{2} \rightarrow \operatorname{gr}\left(O_{Y}\right)$ extends to a surjective homomorphism of graded $A$-Modules [4, III, 2, No. 3, Example 3]:

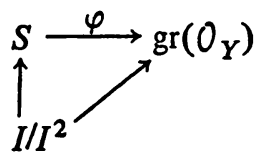

Moreover, there is a canonical surjective homomorphism of graded $A$-Modules [4] $\lambda: \operatorname{gr}\left(O_{Y}\right) \otimes_{A} F / I F \rightarrow \operatorname{gr}(F)$. Thus, we have a canonical surjective homomorphism of graded $A$-Modules $\alpha: S \otimes_{A} F / I F \rightarrow \operatorname{gr}(F)$, by letting $\alpha=\varphi \otimes 1_{F / I F \circ \lambda}$. This defines a graded $S$-Module structure on $\operatorname{gr}(F)$. Moreover, $\operatorname{gr}(F)$ is generated over $S$ by its component of degree zero, I/IF .

Lemma 2. Let $E$ be a coherent graded S-Module. Then there exists an integer $n \geqslant 0$, such that $E(n)$ is generated by $E(n)_{0}=E_{n}$; i.e., such that the canonical homomorphism of $S$-Modules $S \otimes_{O_{Y}} E_{n} \rightarrow E(n)$ is surjective.

Proof. Let $x_{1}, \ldots, x_{s}$ and $e_{1}, \ldots, e_{t}$ generate $I / I^{2}$ and $E$ respectively. Let $n_{j}=\operatorname{Sup}\left(\operatorname{deg}\left(e_{j}\right)\right)-\operatorname{deg}\left(e_{j}\right)$. Any homogeneous element of degree $k \geqslant n$ in $E$ looks like a linear combination of terms

$$
x_{i_{1}} \cdot \ldots \cdot x_{j_{k_{j}}} \cdot e_{j}=\left(x_{i_{1}} \cdot \ldots \cdot x_{i_{n_{j}}} \cdot e_{j}\right)\left(x_{i_{n_{j}+1}} \cdot \ldots \cdot x_{i_{k_{j}}}\right) \text {, }
$$

where $x_{i_{1}} \cdot \ldots \cdot x_{i_{n_{j}}} \cdot e_{j}$ is a member of $E_{n}$. Hence, $E(n)$ is generated by $E_{n}$. This proves Lemma 2 .

Returning to the proof of Proposition 1, let $\left(n_{0}, \ldots, n_{k}\right)$ be any sequence of positive integers. Inductively define the graded $S$-Module $F\left[n_{0}, \ldots, n_{k}\right]$ as follows: Let 


$$
F\left[n_{0}\right]=\operatorname{gr}(F),
$$

$$
F\left[n_{0}, n_{1}\right]=\operatorname{Ker}\left\{S \otimes_{O_{Y}} F\left[n_{0}\right]_{n_{1}} \rightarrow F\left[n_{0}\right]\left(n_{1}\right)\right\},
$$

$F\left[n_{0}, \ldots, n_{k}\right]=\operatorname{Ker}\left\{S \otimes_{O_{Y}} F\left[n_{0}, \ldots, n_{k-1}\right]_{n_{k}} \rightarrow F\left[n_{0}, \ldots, n_{k-1}\right]\left(n_{k}\right)\right\}$.

Note. $F\left[n_{0}, \ldots, n_{k}\right]$ does not depend on $n_{0}$.

Let $d=\operatorname{dim} Y$. According to Lemmas 1 and 2, there exists a sequence of positive integers $n_{0}, \ldots, n_{d}$, for which the graded $S$-Modules $F\left[n_{0}, \ldots, n_{k}\right]$ are coherent for $k=0, \ldots, d$. Choose one such sequence of positive integers and let

$$
A_{k}=F\left[n_{0}, \ldots, n_{k}\right], \quad B_{k}=S \otimes_{\mathcal{O}_{Y}} F\left[n_{0}, \ldots, n_{k-1}\right]_{n_{k}}
$$

for $k=0, \ldots, d$. We then have exact sequences

$$
0 \rightarrow A_{k} \rightarrow B_{k} \rightarrow A_{k-1}\left(n_{k}\right) \rightarrow 0
$$

for $k=1, \ldots, d$. By (3), there exists an integer $M>0$ such that $R^{q} f_{*}\left(B_{k}\left(M^{\prime}\right)\right)=0$ for $q>0, k \geqslant 0$ and $M^{\prime} \geqslant M$. Let $T_{k}=M+\Sigma_{j \geqslant k+1} n_{j}$. For any integer $N$, the function $E \rightarrow E(N)$, from the category of graded $S$-Modules into itself, is exact. Hence, we have the exact sequences

$$
0 \rightarrow A_{k}\left(T_{k}\right) \rightarrow B_{k}\left(T_{k}\right) \rightarrow A_{k-1}\left(T_{k}+n_{k}\right) \rightarrow 0
$$

for $k=1, \ldots, d$. Now notice that

$$
A_{k-1}\left(T_{k}+n_{k}\right)=A_{k-1}\left(T_{k-1}\right),
$$

for $k=1, \ldots, d$. Using [13] and the long exact sequences on $R^{q} f_{*}(\cdot)$ from the $d$ exact sequences of (12), we prove by induction on $k$ that

$$
R^{q} f_{*}\left(A_{k}\left(T_{k}\right)\right)=0 \text { for } q>k \text { and } k=0, \ldots, d .
$$

In particular, we have shown that

$$
R^{q} f_{*}\left(A_{0}\left(T_{0}\right)\right)=0 \text { for } q>0 .
$$

This proves Proposition 1, since

$$
A_{0}\left(T_{0}\right)=F\left[n_{0}\right]\left(T_{0}\right)=\operatorname{gr}(F)\left(T_{0}\right)=\bigoplus_{n \geqslant T_{0}} I^{n} F / I^{n+1} F
$$

Remark 1. The converse of Proposition 1 holds when $X$ is complete and $X, Y$ are regular of dimensions $d$ and $d-1$, respectively. 
REMARK 2. In the case where $Y$ is complete and $I / I^{2}$ is a vector bundle, the proof of Proposition 1 is very short. In fact, for any ample vector bundle $E$ (see [8]) and graded $S(E)$-Module of finite type $F, H^{q}\left(Y, F_{k}\right)=0$ for $q>0$ and $k \gg 0$. This is not difficult to show. If $E$ is an ample vector bundle, then $O_{\mathrm{P}(E)}$ is an ample line bundle on $\mathrm{P}(E)$ [8, III, Theorem 1.1]. There is a Leray spectral sequence

$$
E_{2}^{p, q}=H^{p}\left(Y, R^{q} \pi_{*}\left(\widetilde{F} \otimes O_{\mathrm{P}(E)}(k)\right)\right) \Rightarrow H^{p+q}\left(\mathrm{P}(E), \widetilde{F} \otimes O_{\mathrm{P}(E)}(k)\right)
$$

where $\widetilde{F}$ is the coherent sheaf on $\mathrm{P}(E)$ associated to the graded $S$-Module $F$ and $\pi$ is the natural projection map $\pi: \mathrm{P}(E) \rightarrow X[5$, No. 8, 2.5.2]. Using the fact that

$$
R^{q} \pi_{*}\left(\widetilde{F} \otimes O_{\mathrm{P}(E)}(k)\right)=0 \quad \text { for } q>0 \text { and } k \gg 0
$$

$[11, \S 63]$, we see that the spectral sequence (12) degenerates; i.e.

$$
H^{p}\left(Y, \pi_{*}\left(\widetilde{F} \otimes O_{\mathrm{P}(E)}(k)\right)\right) \approx H^{p}\left(\mathrm{P}(E), \widetilde{F} \otimes \mathcal{O}_{\mathrm{P}(E)}(k)\right)
$$

for $p>0$ and $k \gg 0$. By ampleness of $\mathrm{O}_{\mathrm{P}(E)}(1)$, we have

$$
H^{p}\left(\mathrm{P}(E), \widetilde{F} \otimes \mathcal{O}_{\mathrm{P}(E)}(k)\right)=0 \text { for } p>0 \text { and } k \gg 0 .
$$

By assumption, $F$ is a finite type graded $S(E)$-Module. Hence, by $[11, \S 63$, Proposition 3] we have

$$
F_{k} \approx \pi_{*}\left(\widetilde{F} \otimes O_{\mathrm{P}(E)}(k)\right) \text { for } k \gg 0 .
$$

Combining (16), (17) and (18), we have

$$
H^{p}\left(Y, F_{k}\right)=0 \text { for } p>0 \text { and } k \gg 0 .
$$

We should also remark that this argument would push through to give a proof of Proposition 1 for the case where $Y$ is complete and $I / I^{2}$ is arbitrary, provided that the cohomological criterion for ampleness of a vector bundle [8, III, Theorem 1.1] generalizes to arbitrary coherent sheaves. By proof analysis of Hartshorne's theorems on the subject, we would have this desired generalization provided that the natural map $\left[5\right.$, No. 11, 2.1.12] $S^{k}(E) \rightarrow \pi_{*} \mathrm{O}_{\mathrm{P}(E)}(k)$ is an isomorphism for $k \gg 0$.

One of the main results of Artin's paper [1] is that a formal contraction is algebraic [1, Theorem 3.1]. In $\S 6$ of [1], Artin gives conditions for the existence 
of formal contractions. The following theorem is an immediate corollary of 'one of Artin's main results on algebraization, applied to his existence conditions for formal contractions [1, Theorems 3.1 and 6.2].

THEOREM 1 (ARTIN). Let $X$ be an algebraic space, let $Y=V(I)$ be the closed subspace defined by a defining ideal I of $X$, and let $F: Y \rightarrow Y^{\prime}$ be a proper map. Suppose the following two conditions hold:

(i) For every coherent sheaf $F$ on $X$, we have $R^{q} f_{*}\left(I^{n} F / I^{n+1} F\right)=0$ for $n \gg 0$.

(ii) For every $n$, the map of sheaves on $Y, f_{*}\left(O_{X} / I^{n}\right) \times_{f_{*}} \mathrm{O}_{Y} \mathrm{O}_{Y^{\prime}} \rightarrow \mathrm{O}_{Y^{\prime}}$ is surjective.

Then there is an algebraic space $X^{\prime}$, a defining ideal $I^{\prime} \subseteq 0_{X^{\prime}}$ such that $Y^{\prime}=V\left(I^{\prime}\right)$, and a map $\bar{f}: X \rightarrow X^{\prime}$ that induces $f$ on $Y$ while being an isomorphism outside of $Y$.

Here are some corollaries of Proposition 1 and Theorem 1.

COROLlaRY 1. In Theorem 1, condition (i) may be replaced by the stronger condition that for every coherent sheaf $F$ on $Y$,

$$
R^{1} f_{*}\left(S^{n}\left(I / I^{2}\right) \otimes F\right)=0 \text { for } n \gg 0 .
$$

Condition (ii) may be replaced by the stronger condition that

$$
R^{1} f_{*}\left(I^{n} / I^{n+1}\right)=0 \text { for every } n .
$$

Proof. The replacement of condition (i) follows immediately from Proposition 1.

The replacement of condition (ii) implies that the natural map $f_{*}\left(O_{X} / I^{n}\right) \rightarrow$ $f_{*}\left(0_{X} / I^{n-1}\right)$ is surjective for all $n$, since $I^{n} / I^{n+1}$ is the kernel of the natural map $0_{X} / I^{n} \rightarrow O_{X} / I^{n-1}$. Therefore, $f_{*}\left(O_{X} / I^{n}\right) \rightarrow f_{*}\left(O_{X} / I\right) \approx f_{*}\left(O_{Y}\right)$ is surjective for every $n$. Now right exactness of the functor $X_{f_{*} O_{Y}} O_{Y^{\prime}}$ gives us condition (ii).

CoROllary 2. With the notation of Theorem 1, suppose that $Y^{\prime}$ is a point. Then $Y$ can be contracted to a point in $X^{\prime}$, if for every coherent sheaf $F$ on $Y$, $H^{1}\left(Y, S^{n}\left(I / I^{2}\right) \otimes F\right)=0$ for $n \gg 0$. In particular, $Y$ can be contracted to a point if $I / I^{2}$ is an ample vector bundle [8, III, Theorem 1.1].

Proof. When $Y^{\prime}$ is a point, condition (ii) of Theorem 1 is automatically satisfied.

COROLlary 3. With the notation of Theorem 1, suppose that $Y$ is complete and is of codimension one in $X$, that $Y$ is (locally over $Y^{\prime}=\operatorname{Spec} k$ ) isomor- 
phic to projective space $\mathbf{P}_{Y}^{r}$ for some $r$, and that $S^{n}\left(I / I^{2}\right)$ is locally isomorphic to the bundle of hyperplane sections on $\mathrm{P}^{r}$ for some large $n$. Then $Y$ contracts to a point in $X$; i.e., there exist an algebraic space $X^{\prime}$ and a morphism of algebraic spaces $\bar{f}: X \rightarrow X^{\prime}$ that is a contraction on $Y$ and an isomorphism outside of $Y$. Furthermore, if $X$ is regular, then so is $X^{\prime}$.

Proof. When $Y^{\prime}$ is a point, condition (ii) is automatically satisfied. If $S^{n}\left(I / I^{2}\right)$ is locally isomorphic to the bundle of hyperplane sections on $\mathrm{P}^{r}$ for some large $n$, and if $Y$ is complete and of codimension 1 , then $I / I^{2}$ is an ample line bundle $[8, \mathrm{I}, \mathrm{p} .18]$.

Corollaries 1, 2 and 3 compare with the corollaries of Artin's theorem found in [1, Corollaries $6.10-6.12, \mathrm{pp} .124-125]$.

\section{REFERENCES}

1. M. Artin, Algebraization of formal moduli. II. Existence of modifications, Ann. of Math. (2) 91 (1970), 88-135. MR 41 \#5370.

2. The implicit function theorem in algebraic geometry, Algebraic Geometry (Internat. Colloq., Tata Inst. Fund. Res., Bombay, 1968), Oxford Univ. Press, London, 1969, pp. 13-34. MR 41 \#6847.

3. - Algebraic spaces, Whittemore Lectures, Yale University, 1969 (mimeographed notes).

4. N. Bourbaki, Eléments de mathématiques. Fasc. XXVIII. Algèbre commutative. Chap. 3: Graduations, filtrations et topoligies, Actualités Sci. Indust., no. 1293, Hermann, Paris, 1961. MR 30 \#2027.

5. J. Dieudonné and A. Grothendieck, Eléments de géométrie algébrique, Inst. Hautes Études Sci. Publ. Math. Nos. 8, 11 (1960/61). MR 36 \#177b, c.

6. H. Grauert, Über Modifikationen und exzeptionelle analytische Mengen, Math. Ann. 146 (1962), 331-368. MR 25 \#583.

7. R. Hartshorne, Ample vector bundles, Inst. Hautes Études Sci. Publ. Math. No. 29 (1966), 63-94. MR 33 \#1313.

8. - Ample subvarieties of algebraic varieties, Lecture Notes in Math., vol. 156, Springer-Verlag, Berlin, 1970.

9. D. Knutson, Algebraic spaces, Lecture Notes in Math., vol. 203, Springer-Verlag, Berlin and New York, 1971. MR 46 \#1791.

10. J. Mazur, A relative étale topology for imbedded subschemes (to appear).

11. J.-P. Serre, Faisceaux algébriques cohérents, Ann. of Math. (2) 61 (1955), 197278. MR 16, 953.

12. O. Zariski, Algebraic surfaces, Springer, Berlin, 1935.

DEPARTMENT OF MATHEMATICS, MARLBORO COLLEGE, MARLBORO, VERMONT 05344 